\title{
Exploiting Time Diversity of LoRa Networks Through Optimum Message Replication
}

\author{
Arliones Hoeller Jr. ${ }^{* \dagger}$, Richard Demo Souza*, Onel L. Alcaraz López ${ }^{\ddagger}$, \\ Hirley Alves ${ }^{\ddagger}$, Mário de Noronha Neto ${ }^{\dagger}$, Glauber Brante ${ }^{\S}$ \\ *Electrical \& Electron. Eng. Dept. \\ UFSC, Florianópolis, Brazil \\ richard.demo@ufsc.br \\ ${ }^{\S}$ Commun. Syst. Lab \\ UTFPR, Curitiba, Brazil \\ gbrante@utfpr.edu.br
}

\begin{abstract}
LoRA networks have been gaining momentum as an accessible and open solution for massive connectivity in the Internet-of-Things. Besides the popularity, however, academic research has shown that the technology has limitations. In particular to the connectivity argument, it became clear that massive coverage comes at the price of severely reduced duty cycles, and the increased number of users in the network significantly elevates packet loss due to collisions. In this paper, we exploit time diversity to increase the probability of successful packet delivery in LoRA uplink. We build on previous work to model the outage and coverage probabilities of LORA channels and analyze the use of message replication to create signal diversity. We observed that there is an optimum number of message replicas that minimizes outage probability and avoids high collision probability. We validate the proposed model using numerical simulations.
\end{abstract}

Index Terms-Internet-of-Things, LPWAN, LoRa, Communication Diversity

\section{INTRODUCTION}

The Internet-of-Things (IoT) is pushing a paradigm shift in the design of connectivity solutions for smart devices. Nowadays, the community widely accepts that current connectivity solutions like WiFi, Bluetooth, and ZigBee alone cannot cope with the billions of devices expected to integrate the IoT in the forthcoming years [1]. The IoT is emerging as a solution to integrate different communication technologies, each focusing on the requirements of specific applications. The so-called Massive IoT (mIoT) figures within this context as a network scenario with thousands of connected devices running noncritical, low-power, and low-cost applications tolerant to high latency and small data-rates [2]. New communication technology must address such peculiar scenario, and this is the case of Low-Power Wide-Area Network (LPWAN) technologies like LORAWAN, SigFox, NB-IOT, and RPMA [3].

This paper concerns the assessment of the uplink channel of the LORA (Long-Range) technology, which forms the physical layer (PHY) of the LORAWAN protocol stack [4]. Although LORA is in fast-paced adoption, reports on deployments with large numbers of stations are yet to come out, making their performance and capacity models still an open problem. Recent related work has sought to assess the performance of
LoRA networks using both analytic modelings [5]-[7] and real measurements [8]-[13].

Analytic models have been proposed for a variety of scenarios and communication phenomena. Bor et al. [5] experimentally observed the capture effect of LORA and modeled the capacity of such networks, concluding that LoRA networks with one gateway and conservative operational parameters do not scale well, while networks with dynamic adaptation of operating parameters or multiple gateways scale better. Georgiou and Raza [6] propose an analytic model for LoRA coverage probability that takes into account Rayleigh fading, showing that LORA networks are sensitive to network usage. Pop et al. [7] evaluated the impact of the LoRAWAN downlink on the uplink goodput and coverage probability. They verified that the downlink becomes unable to deliver several acknowledgment packets if too many end-devices request delivery confirmation. Concerning the modeling of communication fading, only Georgiou and Raza [6] and Pop et al. [7] take this impairment into account.

Several works have used measurements to evaluate the performance of LoRA networks. Petäjäjärvi et al. [8] analyze Doppler robustness, scalability, and coverage of LoRA networks and report the experimental validation of such metrics in terrestrial and water environments for static and mobile nodes. There are similar measurement reports in other environments, including a university campus [9], indoor applications [10], industry [11], dense cities downtown [12], and rural areas [13]. These measurements show interesting results, however, none of them used a large numbers of nodes and, thus, it is impossible to use the results to validate dense network models.

As reports on the performance of LORA and other LPWAN started to reveal the limitations of such networks, a few techniques were proposed to enhance the performance of LORA [14]-[16] and other LPWAN technologies [17]-[19].

Cuomo et al. [14] suggest algorithms to replace LoRAWAN adaptive data rate strategy. The algorithms do not base the spreading factor (SF) configuration only on distance and received power, but also take into account the number of connected devices, equalizing the time-on-air (ToA) of packets in each SF. Bor and Roedig [15] explore LoRA configuration 
parameters of SF, bandwidth, coding rate and transmit power and propose an optimization problem that minimizes energy spent on data transmission while meeting required communication performance. Qin and McCann [16] approach the optimization of LPWANs efficiency from a resource allocation perspective. They use game theory to derive an algorithm that allows network nodes to decide which channel and SF to use and, for each channel/SF group, the optimal transmit power maximizing data extraction rate.

Mo et al. [17] investigated the optimum number of message replicas in UNB/SIGFoX networks. Their work takes into account specific aspects of SIGFOX networks, such as the random frequency and time multiple access MAC algorithm. Moreover, the work neglects the effect of fading. Song et al. [18] consider the macro reception diversity of long-range ALOHA networks, where augmented spatial diversity arises from allowing several base stations to receive the same packet. Their model takes into account Rayleigh fading, shadowing and the capture effect to derive closed-form formulae for packet loss. Magrin et al. [19] built a simulation model with which they showed that LoRA networks support a large number of nodes and maintain reasonable network quality if several gateways are carefully placed.

In this paper, we analyze and validate the behavior of LORA networks using message replication to exploit time diversity [20] and maximize network performance. Network performance is modeled and analyzed regarding their outage and coverage probabilities. We follow the work of [6] and extend it to incorporate the proposed technique. Our work differs from [17] because that work considers SIGFOX networks where each transmission uses a random central frequency an assumption that changes the collision model. Moreover, our work takes fading into account, while [17] does not. By analyzing the obtained simulation results, we were able to formulate an optimization problem that finds the optimum number of message replications for a set of network configuration parameters and different network density (number of nodes and their duty cycles).

\section{LORA NETWORKS}

LoRA is a proprietary sub-GHz chirp spread spectrum modulation technique optimized for long-range applications and low power consumption at a low transmission rate [4], which enables the LPWAN paradigm. The technology allows the use of variable transmit rates with constant bandwidth using different orthogonal SFs. The variable data rate characteristic allows optimizing applications according to range, robustness or energy consumption. Using different SFs, several devices can transmit data at the same time, using the same channel frequency without relevant degradation of the received signal.

LORA modulation depends, basically, on three parameters [21]: bandwidth $(B)$, usually set to $125 \mathrm{kHz}$ or $250 \mathrm{kHz}$ for uplink and $500 \mathrm{kHz}$ for downlink; spreading factor (SF), denoted in the equations by $\mathbb{S} \in\{7, \cdots, 12\}$; and the forward error correction rate (FEC), varying from $\frac{4}{8}$ to $\frac{4}{5}$. It is possible to extract from these parameters the packet Timeon-Air (ToA), the receiver sensitivity and the required signal to noise ratio (SNR) for successful detection in the absence of interference. Table I shows the relation among LoRA parameters for a payload of 9 bytes at $B=125 \mathrm{kHz}$ with CRC and Header Mode enabled. It is possible to observe that ToA grows exponentially with $\mathrm{SF}$, reducing the bit rate and increasing the sensitivity, allowing higher coverage.

Table I

LORA UPLINK CHARACTERISTICS CONSIDERING PACKETS OF 9 BYTES AT $B=125 \mathrm{KHz}$ WITH CRC AND HEAdER MOdE ENABLED.

\begin{tabular}{ccccc}
\hline SF & $\begin{array}{c}\text { Time-on-Air } \\
(\mathbf{m s})\end{array}$ & $\begin{array}{c}\boldsymbol{R}_{\boldsymbol{b}} \\
(\mathbf{k b p s})\end{array}$ & $\begin{array}{c}\text { Receiver Sensitivity } \\
(\mathbf{d B m})\end{array}$ & $\begin{array}{c}\text { SNR threshold } \\
\boldsymbol{q}_{\mathbb{S}}(\mathbf{d B})\end{array}$ \\
\hline 7 & 41.22 & 5.47 & -123 & -6 \\
8 & 72.19 & 3.12 & -126 & -9 \\
9 & 144.38 & 1.76 & -129 & -12 \\
10 & 247.81 & 0.98 & -132 & -15 \\
11 & 495.62 & 0.54 & -134.5 & -17.5 \\
12 & 991.23 & 0.29 & -137 & -20 \\
\hline
\end{tabular}

The implementation of LoRA physical layer is agnostic of higher layers. The most used protocol stack featuring a LoRA PHY is LoRAWAN, which implements a star topology and defines three device types: the end-devices (nodes), connected through a single-hop to one or more gateways, which in turn are connected via an IP network to a network server. LORAWAN MAC is performed using unslotted ALOHA.

LoRA gateways can process up to nine channels in parallel, combining different sub-bands ${ }^{1}$ and SFs [1]. Besides that, LORA features the capture effect, making it possible to recover a LORA signal when two or more signals are received simultaneously, using the same frequency and the same SF, provided that the desired signal is at least $6 \mathrm{~dB}$ stronger [5].

\section{SYSTEM MODEL}

Following [6], consider that, on average, $\bar{N}$ nodes are uniformly distributed around a gateway in a circular region of radius $R$ and area $V=\pi R^{2}$. In the coordinate system presented in Figure 1, the gateway is at the origin, and the nodes are deployed uniformly at random in the region $V \subseteq \mathbb{R}^{2}$. This distribution is described by an inhomogeneous Poisson point process (PPP) with density $\rho>0$ in $V$ and 0 otherwise. In this scenario, $d_{i}$ is the Euclidean distance between the gateway and the $i$-th node. Devices transmit in the uplink at random using the ALOHA protocol, obeying a duty cycle $p_{0}$. SF is assigned according to the distance from the gateway $d_{i}$, increasing every $2 \mathrm{~km}$, and all nodes use the same transmit power $\mathcal{P}_{i}$.

The uplink model considers that both path loss attenuation $g\left(d_{i}\right)$ and Rayleigh fading $h_{i}$ affect the received signal. Path loss follows the Friis transmission equation $g\left(d_{i}\right)=\left(\frac{\lambda}{4 \pi d_{i}}\right)^{\eta}$, where $\lambda$ is the wavelength, and $\eta \geq 2$ is the path loss exponent. Rayleigh fading is a zero-mean, independent, circularlysymmetric complex Gaussian random variable with unit variance. Given a signal $s_{1}$ transmitted by a LoRA node, the

\footnotetext{
${ }^{1} \mathrm{~A}$ LoRA sub-band is a set of frequency channels.
} 


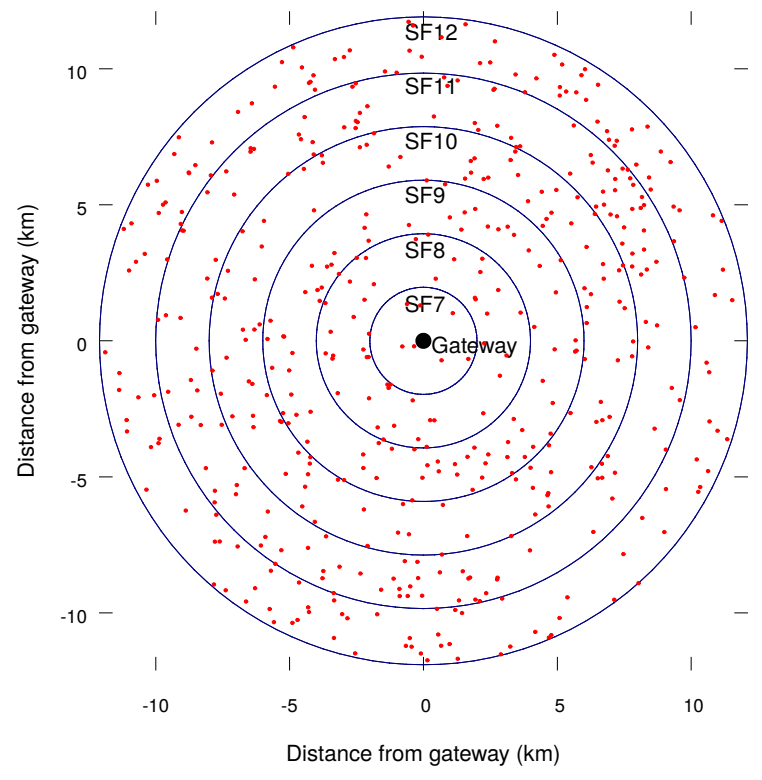

Figure 1. $\quad \bar{N}=500$ nodes uniformly distributed in a circular area of radius $R=12000 \mathrm{~m}$ around the gateway and with increasing SF every $2000 \mathrm{~m}$.

received signal at the gateway, $r_{1}$, is the sum of the attenuated $s_{1}$, interference and noise. It can be expressed as

$$
r_{1}=g\left(d_{1}\right) h_{1} s_{1}+\sum_{k=2}^{N} \chi_{k}^{\mathbb{S}} g\left(d_{k}\right) h_{k} s_{k}+n
$$

where $\chi_{k}^{\mathbb{S}}$ is 1 if the $k$-th node is transmitting in the same SF of the desired signal at a given time or 0 otherwise, and $n$ is additive white Gaussian noise (AWGN) with zero mean and variance $\mathcal{N}=-174+F+10 \log (B) \mathrm{dBm}$. $F$ is the receiver noise figure, and $B$ is the channel bandwidth.

The outage probability model follows [6], where the authors consider that outage occurs if either there is no connection between a node and the gateway or if there is a collision.

\section{A. Outage Condition 1: Disconnection}

First, we consider the connection probability, which depends on the communication distance. A node is assumed not connected to the gateway if the SNR of the received signal is below the reception threshold that allows successful detection in the absence of interference. Receiver sensitivity is different for each SF, what results in different SNR reception thresholds $\left(q_{\mathbb{S}}\right)$, as shown in Table I. The connection probability is thus

$$
H_{1}=\mathbb{P}\left[\operatorname{SNR} \geq q_{\mathbb{S}} \mid d_{1}\right],
$$

where $q_{\mathbb{S}}$ is the SNR reception threshold for the SF $(\mathbb{S})$, and $d_{1}$ is the distance between the desired node and the gateway.

Since we assume Rayleigh fading, the instantaneous SNR is exponentially distributed [20], and therefore

$$
H_{1}=\mathbb{P}\left[\left|h_{1}\right|^{2} \geq \frac{\mathcal{N} q_{\mathbb{S}}}{\mathcal{P}_{1} g\left(d_{1}\right)}\right]=\exp \left(-\frac{\mathcal{N} q_{\mathbb{S}}}{\mathcal{P}_{1} g\left(d_{1}\right)}\right) .
$$

\section{B. Outage Condition 2: Collision}

LoRA exhibits the capture effect [5] because it is a form of frequency modulation, allowing stronger signals to suppress simultaneously received weaker signals. In LoRA, a collision only takes place if the power difference between the desired signal and any other simultaneously received signal using the same SF and frequency channel is less than $6 \mathrm{~dB}$. To evaluate this condition, one must find the strongest interfering node

$$
k^{*}=\arg \max _{k>1}\left\{\mathcal{P}_{k} \chi_{k}^{\mathbb{S}}\left|h_{k}\right|^{2} g\left(d_{k}\right)\right\} .
$$

Once $k^{*}$ is found, the probability that no collision occurs or that the strongest interfering signal is at least $6 \mathrm{~dB}$ below the desired one, termed here as capture probability, is

$$
\begin{aligned}
Q_{1} & =\mathbb{P}\left[\frac{\left|h_{1}\right|^{2} g\left(d_{1}\right)}{\left|h_{k^{*}}\right|^{2} g\left(d_{k^{*}}\right)} \geq 4 \mid d_{1}\right] \\
& =\mathbb{E}_{\left|h_{1}\right|^{2}}\left[\mathbb{P}\left[X_{k^{*}}<\left.\frac{\left|h_{1}\right|^{2} g\left(d_{1}\right)}{4}|| h_{1}\right|^{2}, d_{1}\right]\right] .
\end{aligned}
$$

The probability above depends on the cumulative probability distribution (CDF) of $X_{k^{*}}=\left|h_{k^{*}}\right|^{2} g\left(d_{k^{*}}\right)$, which is derived in [6] and denoted $F_{X_{k^{*}}}$. Thus

$$
Q_{1}=\mathbb{E}_{\left|h_{1}\right|^{2}}\left[F_{X_{k^{*}}}\left(\frac{\left|h_{1}\right|^{2} g\left(d_{1}\right)}{4}\right)\right]=\int_{0}^{\infty} e^{-z} F_{X_{k^{*}}}\left(\frac{z g\left(d_{1}\right)}{4}\right) \mathrm{d} z \text {. }
$$

\section{Coverage Probability}

The coverage probability is the probability that the selected node is in coverage, i.e., it can successfully communicate considering both the connection and collision outage conditions above. The coverage probability of the desired node is thus merely the product $H_{1} Q_{1}$.

Moreover, the average coverage probability $\wp_{c}$ can be obtained by de-conditioning on the position of the desired node achieved by averaging in the network area,

$$
\wp_{c}=\frac{2}{R^{2}} \int_{0}^{R} H_{1}\left(d_{1}\right) Q_{1}\left(d_{1}\right) d_{1} \mathrm{~d} d_{1} .
$$

The remaining of this paper builds on the above model initially presented in [6] by including the required changes to take into account the effects of time diversity.

Additionally, different than [6], in this paper we also consider the average coverage probability per SF (or per annulus). The coverage probability of a node in the annulus $i$ is

$$
\wp_{c, i}=\frac{2}{\left(l_{i+1}-l_{i}\right)^{2}} \int_{l_{i}}^{l_{i+1}} H_{1}\left(d_{1}\right) Q_{1}\left(d_{1}\right)\left(d_{1}-l_{i}\right) \mathrm{d} d_{1},
$$

where $l_{i}$ and $l_{i+1}$ are, respectively, the inner and outer radii of the $i$-th annulus, as illustrated in Figure 1.

\section{Message Replications - Time Diversity}

Many systems increase network reliability by using some form of automatic repeat request (ARQ) technique, in which receivers automatically request the re-transmission of lost packets. Although LoRA systems can use such methods, a recent study [7] showed that LoRA downlink cannot support 
the high demand of confirmation packets generated by nodes in a dense network, making ARQ an unreliable feature. To increase network reliability, however, other techniques can be applied, being message replication one of them.

Now we analyze the effects of message replication in LORA networks, where each information message is transmitted $M$ times within the same time interval when message replication is not in use. Therefore, with message replication, there is an $M$-fold increase in the network duty cycle. Such technique increases the temporal diversity of the desired signal, thus making it much more likely that at least one message copy arrives at the gateway. The increase in the connectivity success probability is dependent on the number of replications $M$.

First, consider the extension of the connection probability, denoted by $H_{1, M}$. In this case, a connection outage only occurs if all $M$ message copies are lost, therefore

$$
H_{1, M}=1-\left(1-H_{1}\right)^{M},
$$

where $\left|h_{1}\right|^{2}$ is assumed independent among replications.

Similar changes take place in the analysis of the capture probability. In this case, (5) can be rewritten to take into account the probability that at least one of the message copies is successfully received,

$$
Q_{1, M}=1-\left(1-Q_{1}\right)^{M} .
$$

It is important to highlight that the derivation of $F_{X_{k^{*}}}$ in [6], required for evaluating $Q_{1}$, defines the number of interfering nodes as a Poisson distribution with mean $v=p_{0} \rho\left|\hat{V}\left(d_{1}\right)\right|$, where $\hat{V}\left(d_{1}\right)$ is the area of the annulus of the desired node. Thus, it is necessary to proportionally increase the mean of the Poisson distribution by making $v=\left(M p_{0}\right) \rho\left|\hat{V}\left(d_{1}\right)\right|$ because the message copies increase the channel usage.

Varying $M$, we observed that the average coverage probability $\left(\wp_{c, i}\right)$ show an optimum point within the bounds of reasonable values of $M$. Thus, we define the following Integer Linear Program (ILP) optimization problem to find the optimum integer number of message copies $M^{*}$ for each $\mathrm{SF}$ in a given network configuration. The ILP is

$$
\begin{aligned}
& M^{*}(i)=\arg \max _{M \in \mathbb{Z}} \wp_{c, i}= \\
& \arg \max _{M \in \mathbb{Z}}\left[\frac{2}{\left(l_{i+1}-l_{i}\right)^{2}} \int_{l_{i}}^{l_{i+1}} H_{1, M}(d 1) Q_{1, M}(d 1)\left(d_{1}-l_{i}\right) \mathrm{d} d_{1}\right] \\
& \text { subject to: } 1 \leq M \leq M_{\max },
\end{aligned}
$$

where $i$ is the annulus (and, thus, SF) under consideration and $M_{\max }$ is the highest value considered for $M^{*}$. In this work, we solved this ILP by enumerating $\wp_{c, i}$ for all possible values of $M$, since the set of possible values of $M$ is small.

\section{NumericAl RESUlts}

This section evaluates the proposed model using numerical simulations. In the figures, solid lines represent theoretical probabilities while points are the results of Monte Carlo simulations. Each simulation point is the averaged result of $10^{5}$ random deployment scenarios. Results are shown for $p_{0}=0.5 \%$, which is equivalent to 18.1 messages per hour
Table II

OPTIMUM VALUES OF $M$ AND THE RESPECTIVE AVERAGE COVERAGE PROBABILITY $\wp_{c}$ FOR EACH SF AND THE WHOLE NETWORK $\left(p_{0}=0.5 \%\right.$ AND $\bar{N}=500$ )

\begin{tabular}{cccccccc}
\hline $\mathbb{S}$ & $\mathbf{7}$ & $\mathbf{8}$ & $\mathbf{9}$ & $\mathbf{1 0}$ & $\mathbf{1 1}$ & $\mathbf{1 2}$ & Network \\
\hline$M^{*}$ & 8 & 5 & 4 & 3 & 3 & 2 & $2,3,4,5,8$ \\
& $94.9 \%$ & $89.7 \%$ & $74.4 \%$ & $58.0 \%$ & $45.6 \%$ & $37.2 \%$ & $59.7 \%$ \\
\hline
\end{tabular}

with $\mathbb{S}=12$, or 436.7 messages per hour with $\mathbb{S}=7$. This is a worst-case scenario since, in practice, LORA applications are expected to operate with duty cycles below $0.1 \%$, and rarely above $0.5 \%$.Moreover, following [6], all results presented in this paper consider $F=6 \mathrm{dBm}, \eta=2.75, \lambda=c / f \mathrm{~m}$, $f=868 \mathrm{MHz}, B=125 \mathrm{kHz}$, which are typical configurations for sub-urban scenarios following European regulations.

Figure 2 shows the performance of the baseline model $(M=1)$ and the message replication technique $(M=\{3,6\})$ with an average number of nodes of $\bar{N}=500$. The plots show the connection probability $H_{1}$, capture probability $Q_{1}$, and coverage probability $H_{1} Q_{1}$ in separated curves. As can be seen, $Q_{1}$ is the primary responsible for network quality degradation. That happens because $Q_{1}$ takes interference into account, which is substantially affected by increasing number of message copies. It is possible to note that $Q_{1}$ decreases with $\mathrm{SF}$, what is expected behavior and happens due to the combination of two factors. The first one is that ToA increases with SF (see Table I). The second one is that, because outer annuli are bigger than inner annuli, and nodes are distributed uniformly in the circular region around the gateway, the scenario has more nodes using higher SFs. Figures $2 \mathrm{~b}$ and $2 \mathrm{c}$ show, as expected, that message replication has a substantial positive impact on $H_{1, M}$ because $M$ decreases this outage condition exponentially. For $Q_{1, M}$, however, the positive impact only exists as long as the message copies do not flood the network, reaching a point where the number of collisions is too high. Moreover, message replication performs better in lower SF than higher SF, what happens because ToA nearly doubles for each SF increase, making it faster to flood the network with message replications when using higher SFs.

Figure 3 shows the average coverage probability $\wp_{c, i}$ fore each SF, for a varying number of message copies $M$. It is possible to observe that the best number of message replications is different for each SF configuration. Table II summarizes, for each SF and the entire network, the optimum number of message copies $\left(M^{*}\right)$ and the corresponding average coverage probability for the annulus that is using that SF. The last column of the table shows the average coverage probability of the network if each node uses the $M^{*}$ computed for its SF. Note that the optimum number of message copies is a decreasing function of the SF, what makes sense since message replications increase the network usage.

\section{CONCLUSION}

In this paper, we analyzed the use of time diversity to enhance the uplink performance in LoRA networks. We imple- 
(a) $\mathrm{p}_{0}=0.5 \%, \overline{\mathrm{N}}=500, \mathrm{M}=1$

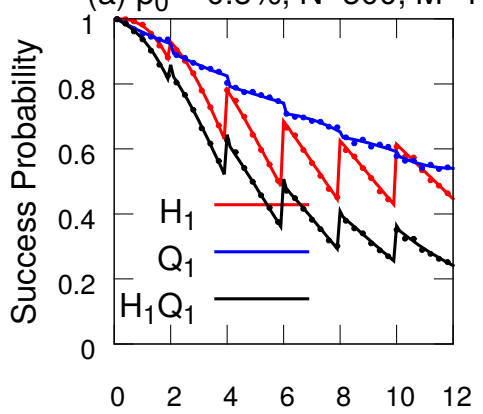

Distance from gateway $(\mathrm{km})$

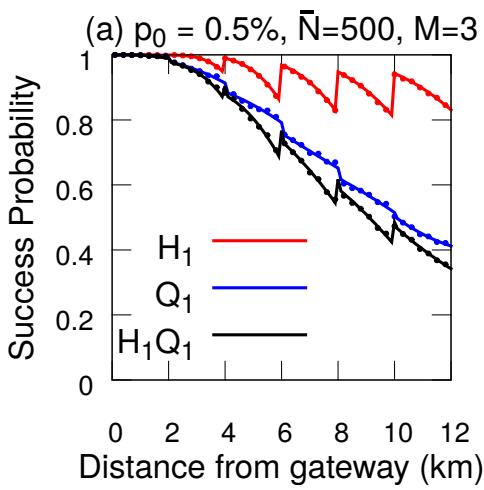

Figure 2. Impact of message replication in LoRA uplink, considering an average number of nodes $\bar{N}=500$, a duty cycle of $p_{0}=0.5 \%$, and different numbers of message copies $M$.

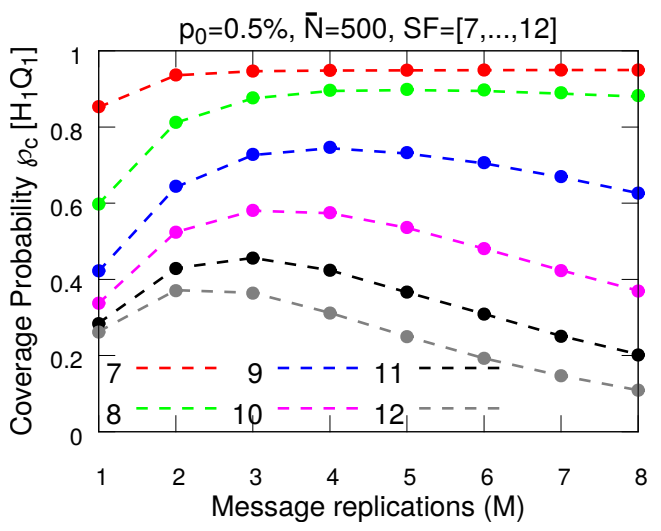

Figure 3. Coverage probabilities of LoRA network with average number of nodes $\bar{N}=500$ and duty cycle $p_{0}=0.5 \%$ for each SF $(\mathbb{S} \in\{7, \cdots, 12\})$.

mented time diversity through message replication. Theoretical modeling and computer simulations were used to investigate the proposed method. We observed that there is an optimal number of copies to be employed for each network configuration and each spreading factor. Different spreading factors tend to feature a different optimum number of message copies that minimizes outage and, in turn, maximizes coverage. Results show that replication is especially useful in low-density networks.

Future work can consider other types of diversity, such as spatial and spectrum diversity. Also, future work may explore the optimization of message replication taking into account energy efficiency, as well as the allocation of different $M$ for different users in a way to favor nodes that require a larger Quality-of-Service (QoS).

\section{REFERENCES}

[1] M. Centenaro, L. Vangelista, A. Zanella, and M. Zorzi, "Long-range commun. in unlicensed bands: the rising stars in the IoT and smart city scenarios," IEEE Wireless Commun., vol. 23, no. 5, Oct 2016.
[2] Ericsson AB, "Cellular netw. for massive IoT - enabling low power wide area appl." Ericsson AB, Tech. Rep. UEN 284 23-3278, Jan 2016.

[3] S. Andreev, O. Galinina, A. Pyattaev, M. Gerasimenko, T. Tirronen, J. Torsner, J. Sachs, M. Dohler, and Y. Koucheryavy, "Understanding the IoT connectivity landscape: a contemporary M2M radio technology roadmap," IEEE Commun. Mag., vol. 53, no. 9, Sept 2015.

[4] LoRa Alliance. (2018, Mar) Website. [Online]. Available: http://www.lora-alliance.org

[5] M. Bor, U. Roedig, T. Voigt, and J. M. Alonso, "Do LoRa low-power wide-area netw. scale?" in Proc. of the 19th ACM Int. Conf. on Modeling, Anal. and Simulation of Wireless Mobile Syst. (MSWiM), Malta, 2016.

[6] O. Georgiou and U. Raza, "Low power wide area netw. anal.: Can LoRa scale?" IEEE Wireless Commun. Lett., vol. 6, no. 2, Apr 2017.

[7] A.-I. Pop, U. Raza, P. Kulkarni, and M. Sooriyabandara, "Does bidirectional traffic do more harm than good in LoRaWAN based LPWA networks?" in 2017 IEEE Global Commun. Conf. (GLOBECOM), 2017.

[8] J. Petäjäjärvi, K. Mikhaylov, M. Pettissalo, J. Janhunen, and J. Iinatti, "Performance of a low-power wide-area netw. based on LoRa technol.: Doppler robustness, scalability, and coverage," Int. J. of Distributed Sensor Netw., vol. 13, no. 3, 2017.

[9] S.-Y. Wang, Y.-R. Chen, T.-Y. Chen, C.-H. Chang, Y.-H. Cheng, C.-C. Hsu, and Y.-B. Lin, "Performance of LoRa-based IoT appl. on campus," in IEEE 86th Veh. Technol. Conf. (VTC-Fall), Sept 2017, pp. 1-6.

[10] P. Neumann, J. Montavont, and T. Noel, "Indoor deploym. of low-power wide area netw. (LPWAN): A LoRaWAN case study," in Int. Conf. on Wireless and Mobile Comp., Netw. and Commun. (WiMOB), 2016.

[11] L. Angrisani, P. Arpaia, F. Bonavolonta, M. Conti, and A. Liccardo, "LoRa protocol performance assessment in critical noise conditions," in IEEE 3rd Int. For. on Res. and Technol. for Soc. and Ind.(RTSI), 2017.

[12] J. Pascal, B. Stefan, F. Liedmann, and C. Wietfeld, "Urban channel models for smart city IoT-networks based on empirical measurements of LoRa-links at 433 and $868 \mathrm{mhz}$," in IEEE 28th Annu. Int. Symp. on Personal, Indoor, and Mobile Radio Commun. (PIMRC), no. Cn I, 2017.

[13] R. Oliveira, L. Guardalben, and S. Sargento, "Long range commun. in urban and rural environ." in IEEE Symp. on Comp. and Commun., 2017.

[14] F. Cuomo, M. Campo, and A. Caponi, "EXPLoRa: Extending the perf. of lora by suitable spreading factor allocations," in IEEE 13th Int. Conf. on Wireless and Mobile Comp., Netw. and Commun. (WiMOB), 2017.

[15] M. Bor and U. Roedig, "LoRa transmission parameter selection," in 13th Int. Conf. on Distributed Computing in Sensor Syst. (DCOSS), 2017.

[16] Z. Qin and J. A. Mccann, "Resource effic. in low-power wide-area netw. for IoT appl." in IEEE Global Commun. Conf. (GLOBECOM), 2017.

[17] Y. Mo, M.-T. Do, C. Goursaud, and J.-M. Gorce, "Optimization of the predefined number of replications in a UNB-based IoT netw." in Wireless Days (WD), Mar 2016.

[18] Q. Song, X. Lagrange, and L. Nuaymi, "Eval. of macro diversity gain in long range ALOHA netw." IEEE Commun. Lett., vol. 21, no. 11, 2017.

[19] D. Magrin, M. Centenaro, and L. Vangelista, "Performance eval. of LoRa netw. in a smart city scenario," in IEEE Int. Conf. on Commun., 2017.

[20] A. Goldsmith, Wireless Commun. USA: Cambridge Univ. Press, 2005.

[21] Semtech, AN120.22 LoRa Modulation Basics(Appl.Note), Mar 2015. 